\title{
Finding Maximum Edge Bicliques in Convex Bipartite Graphs ${ }^{\star}$
}

\author{
Doron Nussbaum ${ }^{1}$, Shuye $\mathrm{Pu}^{2}$, Jörg-Rüdiger Sack ${ }^{1}$, Takeaki Uno ${ }^{3}$, and \\ Hamid Zarrabi-Zadeh ${ }^{1}$ \\ 1 School of Computer Science, Carleton University, Ottawa, Ontario K1S 5B6, \\ Canada. Email: \{nussbaum, sack, zarrabi\}@scs.carleton.ca \\ 2 Program in Molecular Structure and Function, Hospital for Sick Children, \\ 555 University Avenue, Toronto, Ontario M5G 1X8, Canada. \\ Email: shuyepu@sickkids.ca \\ 3 National Institute of Informatics, 2-1-2 Hitotsubashi, Chiyoda-ku, Tokyo 101-8430, \\ Japan. Email: uno@nii.ac.jp
}

\begin{abstract}
A bipartite graph $G=(A, B, E)$ is convex on $B$ if there exists an ordering of the vertices of $B$ such that for any vertex $v \in A$, vertices adjacent to $v$ are consecutive in $B$. A complete bipartite subgraph of a graph $G$ is called a biclique of $G$. Motivated by an application to analyzing DNA microarray data, we study the problem of finding maximum edge bicliques in convex bipartite graphs. Given a bipartite graph $G=(A, B, E)$ which is convex on $B$, we present a new algorithm that computes a maximum edge biclique of $G$ in $O\left(n \log ^{3} n \log \log n\right)$ time and $O(n)$ space, where $n=|A|$. This improves the current $O\left(n^{2}\right)$ time bound available for the problem. We also show that for two special subclasses of convex bipartite graphs, namely for biconvex graphs and bipartite permutation graphs, a maximum edge biclique can be computed in $O(n \alpha(n))$ and $O(n)$ time, respectively, where $n=\min (|A|,|B|)$ and $\alpha(n)$ is the slowly growing inverse of the Ackermann function.
\end{abstract}

\section{Introduction}

DNA Microarray technology has recently become a main stream tool in molecular biology for studying the interaction between genes and conditions. Microarray data is often presented as a two-dimensional matrix, where the rows correspond to genes (or clones, open reading frames, etc.), and columns correspond to test conditions (or samples, treatments, time points, etc.). Each entry $[i, j]$ of the matrix represents an expression level of a given gene $i$ measured under a given condition $j$. Biologists are often capturing the relationships between subsets of genes and subsets of conditions to better understand the biological processes at the cell and the molecular level. For example, biologists are interested in (i) finding a subset of genes that are up-regulated or down-regulated in a coherent

\footnotetext{
* Research supported by NSERC and SUN Microsystems. A preliminary version of this work is to appear in the 16th Annual International Computing and Combinatorics Conference (COCOON 2010).
} 


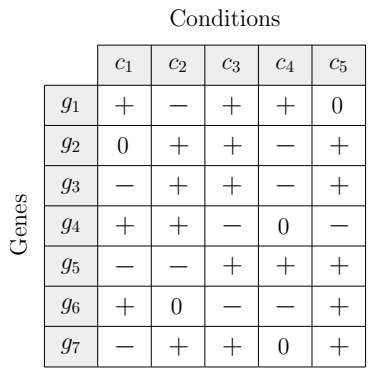

(a)

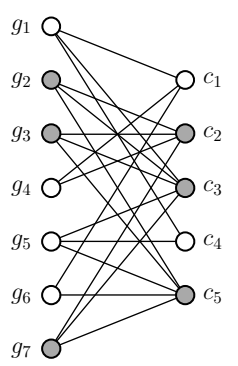

(b)

Fig. 1. (a) An exemplary microarray data matrix. Entries are transformed from numerical values to signs to indicate directions of changes of gene expression levels. Each symbol + or - represents a significant increase or a significant decrease, respectively, while the symbol $\emptyset$ represents no significant change. In this example the subset of genes $\left\{g_{2}, g_{3}, g_{7}\right\}$ and the subset of conditions $\left\{c_{2}, c_{3}, c_{5}\right\}$ form a bicluster. (b) A bipartite graph representing significant increases in the microarray data matrix. Vertices forming a maximum edge biclique in this graph are shown in gray.

fashion under a subset of conditions, or, (ii) finding a subset of conditions (or drugs, diseases) that consistently affect the expression of a subset of genes.

Traditional clustering methods focused on clustering either genes or conditions, but not both, and thus did not provide enough information required by biologists. Biclustering analysis techniques capture the relationship between genes and conditions and have recently become popular tools in bioinformatics [7]. The objectives of biclustering algorithms is to find a subset of genes $\mathcal{G}$ and a subset of conditions $\mathcal{C}$ such that the change in the expression level of each $g \in \mathcal{G}$ with respect to each $c \in \mathcal{C}$ is significant. The general biclustering problem is NP-hard [25]. Several heuristic biclustering algorithms have been reported in the literature, and most of them are based on greedy and probabilistic approaches $[3,7,22,29]$. While these algorithms have technical merits in finding biclusters, they do not guarantee to produce the optimal solution.

A microarray data matrix is composed of a set of genes $\mathcal{G}=\left\{g_{1}, \ldots, g_{n}\right\}$ and a set of conditions $\mathcal{C}=\left\{c_{1}, c_{2}, \ldots, c_{k}\right\}$, such as the one shown in Figure 1(a). This microarray can be modeled as a bipartite graph $G=(A, B, E)$, where each vertex $a_{i} \in A$ represents a gene $g_{i} \in \mathcal{G}$ and each vertex $b_{j} \in B$ represents a condition $c_{j} \in \mathcal{C}$, and two vertices $a_{i} \in A$ and $b_{j} \in B$ are connected, if the expression level of gene $g_{i}$ significantly increases or significantly decreases under condition $c_{j}$ (see Figure 1(b)). A bicluster in this graph corresponds to a bipartite subgraph with two vertex sets $S \subseteq A$ and $T \subseteq B$ such that each vertex in $S$ is connected to each vertex in $T$. Such a complete bipartite subgraph is called a biclique of $G$.

In this paper, we address the problem of finding a maximum bicluster in the expression data, which is equivalent to finding a biclique of maximum edge cardinality in the corresponding bipartite graph. Formally, given a bipartite graph 
$G=(A, B, E)$, the problem is to find a biclique with two vertex sets $S \subseteq A$ and $T \subseteq B$ such that $|S| \times|T|$ is maximized. This problem is called maximum edge biclique, as opposed to the maximum vertex biclique problem in which the objective is to maximize $|S|+|T|$. Besides applications to molecular biology, maximum edge biclique has applications to manufacturing optimization [9], formal concept analysis [14], and conjunctive clustering [24].

While maximum vertex biclique is solvable in polynomial time, the maximum edge biclique problem in general bipartite graphs is known to be NPcomplete [25]. Indeed, it is hard to approximate the maximum edge biclique in general bipartite graphs to within a factor of $n^{\delta}$, for some $\delta>0[12,17]$ (see also [28] for corresponding inapproximability results in weighted version of the problem). However, for special subclasses of bipartite graphs, such as chordal bipartite graphs and convex graphs, polynomial-time algorithms for the maximum edge biclique problem are available using algorithms that enumerate all maximal bicliques in a given graph $[2,10,11,15,19]$.

Convex bipartite graphs introduced by Glover [16] naturally arise in several industrial and scheduling applications, and a number of efficient algorithms have been developed on this graph class for problems such as maximum matching, maximum independent set, and minimum feedback vertex set (see e.g. [6, 20, 21, $26,27])$. A bipartite graph $G=(A, B, E)$ is called convex (on $B)$ if there exists an ordering of the vertices of $B$ such that, for any vertex $v \in A$, vertices adjacent to $v$ are consecutive in $B$. The motivation for studying convex bipartite graphs in the context of biclustering of gene expression data is that a linear ordering of genes exists naturally in several forms, such as chronological ordering in the course of evolution, and spatial ordering on chromosomes.

All the existing algorithms for solving the maximum edge biclique problem are based on enumerating all maximal bicliques in the input graph (see e.g. [2, $11,19])$. It is known that the number of maximal bicliques in a convex bipartite graph with $n$ vertices is $O\left(n^{2}\right)$ [2]. Indeed, it is not hard to construct convex bipartite graphs that have $\Theta\left(\mathrm{n}^{2}\right)$ maximal bicliques. Therefore, the existing algorithms for solving the maximum edge biclique problem have a running time of $\Omega\left(n^{2}\right)$ on convex bipartite graphs.

In this paper, we show that the maximum edge biclique problem can be solved more efficiently on convex bipartite graphs by using a pruning technique that avoids enumerating all maximal bicliques. More precisely, we present a new algorithm that, given a convex bipartite graph $G=(A, B, E)$, computes a maximum edge biclique of $G$ in $O\left(n \log ^{3} n \log \log n\right)$ time and $O(n)$ space, where $n=|A|$. This improves the current $O\left(n^{2}\right)$ time bound currently available for the problem [19]. For two special subclasses of convex bipartite graphs, namely biconvex graphs and bipartite permutation graphs (see definitions in Section 2), we show that a maximum edge biclique can be computed even more efficiently in $O(n \alpha(n))$ and $O(n)$ time, respectively, where $n=\min (|A|,|B|)$, and $\alpha(n)$ is the slowly growing inverse of the Ackermann function. Note that in all these algorithms, we assume that the corresponding ordering on the vertex set that 
defines the convex, biconvex, and bipartite permutation graph is given as part of the input.

The remainder of this paper is organized as follows. Section 2 provides graph theoretical preliminaries. In Section 3, we present an efficient algorithm for solving the maximum edge biclique problem in convex bipartite graphs via a transformation to a geometric problem. Section 4 shows how the maximum edge biclique problem can be solved efficiently in biconvex graphs and bipartite permutation graphs. We conclude the paper with Section 5.

\section{Preliminaries}

A graph $G$ is bipartite, if its set of vertices can be partitioned into two disjoint sets $A$ and $B$ such that every edge of $G$ connects a vertex in $A$ to a vertex in $B$. We denote such a bipartite graph by $G=(A, B, E)$, where $E$ is the set of edges of $G$. A complete bipartite subgraph of a graph $G$ is called a biclique of $G$. Given a biclique $C$ of $G$, we refer to the number of edges in $C$ by the size of $C$, and denote it by $|B|$. Moreover, for a vertex $v$ of $G$, we denote the set of vertices adjacent to $v$ by $N(v)$.

Let $G=(A, B, E)$ be a bipartite graph. An ordering $\prec$ of $B$ has the adjacency property if for every vertex $a \in A, N(a)$ consists of vertices that are consecutive (i.e., form an interval) in the ordering $\prec$ of $B$. A bipartite graph $G=(A, B, E)$ is convex if there is an ordering of $A$ or $B$ that fulfills the adjacency property. Throughout this paper, we always assume that $G$ is convex on $B$. The bipartite graph $G$ is called biconvex (or doubly convex) if it is convex on both $A$ and $B$, i.e., there is an ordering of $A$ and an ordering of $B$ that both fulfill the adjacency property.

Given a bipartite graph $G=(A, B, E)$, an ordering $\prec$ of $B$ has the enclosure property if for every pair of vertices $a, b \in A$ such that $N(a) \subset N(b)$, vertices in $N(b) \backslash N(a)$ occur consecutively in the ordering $\prec$ of $B$. A bipartite graph $G=(A, B, E)$ is bipartite permutation if there is an ordering of $A$ and an ordering of $B$ that both fulfill the adjacency and the enclosure properties. See [5] for alternative definitions of bipartite permutation graphs.

For convex, biconvex, and bipartite permutation graphs, there are linear-time recognition algorithms that output the corresponding orderings on the vertex sets in linear time $[4,18,23]$. Throughout this paper, we assume that in the input graph, vertices of $A$ and $B$ are labeled with integers $1,2, \ldots$ in the order imposed by the corresponding orderings of $A$ and $B$. Figure 2 shows examples of different classes of convex bipartite graphs.

In this paper, we denote by $[a . . b]$ the set of integers that lie between two integers $a$ and $b$, including both. Such a set is called an integer interval. Given an integer interval $I=[a . . b]$, the size of $I$, denoted by $|I|$, is the number of integers, $b-a+1$, contained in $I$. 


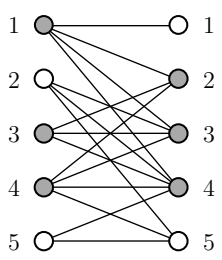

(a)

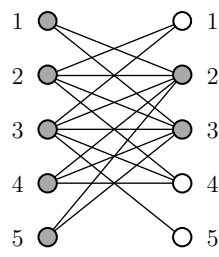

(b)

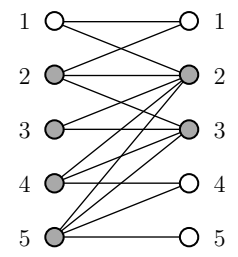

(c)

Fig. 2. (a) A convex bipartite graph. (b) A biconvex graph. (c) A bipartite permutation graph. The shaded vertices in each graph represent a maximum edge biclique.

\section{An Algorithm for Convex Bipartite Graphs}

In this section, we provide an efficient algorithm for the maximum edge biclique problem in convex bipartite graphs. In order to solve the problem, we first transform it from a graph theoretical problem to a geometric problem, and then show how the geometric problem can be solved efficiently.

\subsection{Problem Transformation}

The main problem considered in this paper is the following:

Problem 1 (Maximum Edge Biclique). Given a convex bipartite graph $G=$ $(A, B, E)$ with $|A|=n$ and $|B|=k$, find a biclique of $G$ that has the maximum number of edges.

We transform the maximum edge biclique problem to a variant of the point dominance problem in the plane. Given two points $p, q \in \mathbb{R}^{2}$, we say that $q$ is dominated by $p$ if $q_{x} \leqslant p_{x}$ and $q_{y} \geqslant p_{y}$ (in other words, if $q$ lies in a rectangle whose bottom-right corner is at $p$ ). Let $S$ be a set of $n$ points in the grid $[1 \ldots k] \times$ $[1 . . k]$. We refer to each point of $S$ as a token. For a grid point $(i, j)$, we define the dominance number of $(i, j)$ w.r.t. $S$ to be $\operatorname{Dom}(i, j)=\mid\{(x, y) \in S:(x, y)$ is dominated by $(i, j)\} \mid$, and define the magnitude of $(i, j)$ to be $\operatorname{MAG}(i, j)=$ $\operatorname{DOM}(i, j) \times(j-i+1)$. We call a grid point maximizing $\operatorname{MAG}(i, j)$ a maximum point of the grid.

Problem 2 (Maximum Point). Given a set $S$ of $n$ tokens on a $k \times k$ grid, find a grid point $(i, j)$ that maximizes $\operatorname{MAG}(i, j)$.

Lemma 1. Problem 1 is equivalent to Problem 2.

Proof. By the convexity of $G, N(a)$ is an integer interval in $[1 . . k]$ for each vertex $a \in A$. Let $\pi$ be a function that maps each integer interval $[i . . j] \subseteq[1 \ldots k]$ to a grid point $(i, j)$ on the grid $[1 . . k] \times[1 \ldots k]$. For each vertex $a \in A$, we define $\pi(a) \equiv \pi(N(a))$. Let $S=\{\pi(a): a \in A\}$. We show that finding a maximum edge 


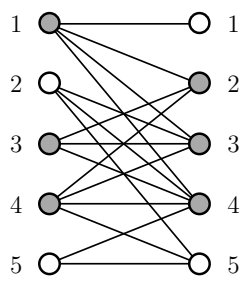

(a)

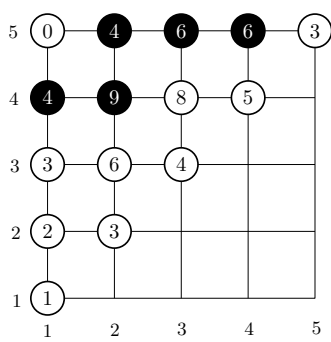

(b)

Fig. 3. (a) A convex bipartite graph. (b) The corresponding grid. Tokens are shown in black. The number inside each grid point denotes its magnitude.

biclique in $G$ is equivalent to solving the maximum point problem on the set $S$ (see Figure 3).

The key observation is that for any pair of integer intervals $I$ and $R, R \subseteq I$ if and only if $\pi(I)$ is dominated by $\pi(R)$. This is because $[i . . j] \subseteq\left[i^{\prime} . . j^{\prime}\right]$ if and only if $i^{\prime} \leqslant i$ and $j^{\prime} \geqslant j$ which means that $\left(i^{\prime}, j^{\prime}\right)$ is dominated by $(i, j)$. Let $R=[i . . j] \subseteq[1 . . k]$ represent a set of consecutive vertices in $B$. Define $A_{R}=\{a \in A: R \subseteq N(a)\}$. Every vertex in $A_{R}$ is connected to every vertex in $R$. Therefore, $A_{R} \times R$ defines a biclique $C_{R}$ of $G$ with $\left|A_{R}\right| \times|R|$ edges. Moreover, $\left|A_{R}\right|=|\{a \in A: R \subseteq N(a)\}|=\mid\{a \in A: \pi(N(a))$ is dominated by $\pi(R)\} \mid=$ $\mid\{a \in A: \pi(a)$ is dominated by $(i, j)\} \mid=\operatorname{Dom}(i, j)$ w.r.t. $S$. Therefore, $\left|C_{R}\right|=$ $\left|A_{R}\right| \times|R|=\operatorname{DOM}(i, j) \times(j-i+1)=\operatorname{MAG}(i, j)$, and thus, finding a clique of maximum size in $G$ is equivalent to finding a grid point with maximum magnitude.

Note that $\operatorname{MAG}(i, j)$ is less than or equal to zero for $j<i$. Therefore, to find a maximum point, we only need to consider grid points $(i, j)$ with $j \geqslant i$.

\subsection{The Algorithm}

Let $S=\left\{\left(x_{1}, y_{1}\right), \ldots,\left(x_{n}, y_{n}\right)\right\}$ be a set of $n$ tokens on a $k \times k$ grid. It is easy to find a maximum point in this grid by a simple scan over the grid points. If the number of tokens is small compared to the grid size, then tokens are sparsely scattered across the grid. An immediate question is whether we can compute the maximum magnitude without necessarily visiting all the grid points. In this section, we answer this question affirmatively by providing an algorithm that finds a maximum point by examining only a small subset (namely, a subquadratic number) of the grid points. We start by the following observation.

Observation 1 If $(x, y)$ is a maximum point, then there are tokens $\left(x_{i}, y_{i}\right)$ and $\left(x_{j}, y_{j}\right)$ in $S$ such that $x_{i}=x$ and $y_{j}=y$.

Proof. Suppose by contradiction that there is no token in $S$ such that $x_{i}=x$. Let $Q \subseteq S$ be the set of tokens dominated by $(x, y)$, and let $q=\left(x^{\prime}, y^{\prime}\right)$ be the 
token with the largest $x$-coordinate in $Q$ (ties are broken arbitrarily). Obviously, $\operatorname{DOM}\left(x^{\prime}, y\right)=\operatorname{DOM}(x, y)$, as there is no token in the rectangle $\left[x^{\prime}+1, x\right] \times[y, k]$. Moreover, $x^{\prime}<x$ by our selection of $q$. Therefore, $\operatorname{DOM}\left(x^{\prime}, y\right) \times\left(y-x^{\prime}+1\right)>$ $\operatorname{DOM}(x, y) \times(y-x+1)$, which means that MAG $\left(x^{\prime}, y\right)>\operatorname{MAG}(x, y)$, contradicting the assumption that $(x, y)$ is a maximum point. Similarly, if there is no token with $y_{j}=y$, we get into a similar contradiction.

Observation 1 enables us to restrict candidates for a maximum point to $\left(x_{i}, y_{j}\right)$, for some $1 \leqslant i, j \leqslant n$. Let $P=\left\{x: x=x_{i}\right.$ for some $\left.i\right\}$ and $Q=$ $\left\{y: y=y_{j}\right.$ for some $\left.j\right\}$. Let $s=|P|$ and $t=|Q|$. We denote the elements in $P$ in increasing order by $\left(p_{1}, \ldots, p_{s}\right)$, and the elements in $Q$ in increasing order by $\left(q_{1}, \ldots, q_{t}\right)$. A maximum point can be now found via a simple scan over the grid points in $P \times Q$ in $O(n+s \times t)=O\left(n^{2}\right)$ time. (Note that this time bound is independent of the size of the original grid, $k$.) In the following, we show that this $O\left(n^{2}\right)$ bound can be further improved, using a novel pruning of the candidate points.

Let $\mathrm{D}(i, j) \equiv \operatorname{DOM}\left(p_{i}, q_{j}\right)$ be the dominance number, and $\mu(i, j) \equiv \operatorname{MAG}\left(p_{i}, q_{j}\right)$ be the magnitude of the grid point $\left(p_{i}, q_{j}\right)$ in our refined grid. The problem is to find a pair $(i, j)$ for which $\mu(i, j)$ is maximum. We define $\operatorname{GAP}\left(i, j, j^{\prime}\right)=\mu\left(i, j^{\prime}\right)-$ $\mu(i, j)$ for $j^{\prime} \geqslant j$. For example, in the grid shown in Figure $4, \operatorname{GAP}(3,5,7)=-4$ and $\operatorname{GAP}(5,5,7)=4$.

Lemma 2. For indices $i<i^{\prime}$ and $j<j^{\prime}, \operatorname{GAP}\left(i, j, j^{\prime}\right) \leqslant \operatorname{GAP}\left(i^{\prime}, j, j^{\prime}\right)$ if there is no token at $(x, y)$ such that $p_{i}<x \leqslant p_{i^{\prime}}$ and $q_{j} \leqslant y<q_{j^{\prime}}$.

Proof. By the assumption of the lemma we have

$$
\mathrm{D}\left(i^{\prime}, j\right)-\mathrm{D}(i, j)=\mathrm{D}\left(i^{\prime}, j^{\prime}\right)-\mathrm{D}\left(i, j^{\prime}\right) .
$$

Observe that

$$
\begin{aligned}
\mu\left(i^{\prime}, j\right)-\mu(i, j) & =\mathrm{D}\left(i^{\prime}, j\right) \times\left(q_{j}-p_{i^{\prime}}+1\right)-\mathrm{D}(i, j) \times\left(q_{j}-p_{i}+1\right) \\
& =\mathrm{D}\left(i^{\prime}, j\right) \times\left(q_{j}-p_{i^{\prime}}+1\right)-\mathrm{D}(i, j) \times\left(q_{j}-p_{i^{\prime}}+p_{i^{\prime}}-p_{i}+1\right) \\
& =\left(\mathrm{D}\left(i^{\prime}, j\right)-\mathrm{D}(i, j)\right) \times\left(q_{j}-p_{i^{\prime}}+1\right)-\mathrm{D}(i, j) \times\left(p_{i^{\prime}}-p_{i}\right) \\
& =\left(\mathrm{D}\left(i^{\prime}, j^{\prime}\right)-\mathrm{D}\left(i, j^{\prime}\right)\right) \times\left(q_{j}-p_{i^{\prime}}+1\right)-\mathrm{D}(i, j) \times\left(p_{i^{\prime}}-p_{i}\right) .
\end{aligned}
$$

Similarly, we have

$$
\mu\left(i^{\prime}, j^{\prime}\right)-\mu\left(i, j^{\prime}\right)=\left(\mathrm{D}\left(i^{\prime}, j^{\prime}\right)-\mathrm{D}\left(i, j^{\prime}\right)\right) \times\left(q_{j^{\prime}}-p_{i^{\prime}}+1\right)-\mathrm{D}\left(i, j^{\prime}\right) \times\left(p_{i^{\prime}}-p_{i}\right) .
$$

Therefore,

$$
\begin{aligned}
\operatorname{GAP}\left(i^{\prime}, j, j^{\prime}\right)- & \operatorname{GAP}\left(i, j, j^{\prime}\right) \\
= & \left(\mu\left(i^{\prime}, j^{\prime}\right)-\mu\left(i^{\prime}, j\right)\right)-\left(\mu\left(i, j^{\prime}\right)-\mu(i, j)\right) \\
= & \left(\mu\left(i^{\prime}, j^{\prime}\right)-\mu\left(i, j^{\prime}\right)\right)-\left(\mu\left(i^{\prime}, j\right)-\mu(i, j)\right) \\
= & {\left[\left(\mathrm{D}\left(i^{\prime}, j^{\prime}\right)-\mathrm{D}\left(i, j^{\prime}\right)\right) \times\left(q_{j^{\prime}}-p_{i^{\prime}}+1\right)-\mathrm{D}\left(i, j^{\prime}\right) \times\left(p_{i^{\prime}}-p_{i}\right)\right] } \\
& \quad-\left[\left(\mathrm{D}\left(i^{\prime}, j^{\prime}\right)-\mathrm{D}\left(i, j^{\prime}\right)\right) \times\left(q_{j}-p_{i^{\prime}}+1\right)-\mathrm{D}(i, j) \times\left(p_{i^{\prime}}-p_{i}\right)\right] \\
= & \left(\mathrm{D}\left(i^{\prime}, j^{\prime}\right)-\mathrm{D}\left(i, j^{\prime}\right)\right) \times\left(q_{j^{\prime}}-q_{j}\right)+\left(\mathrm{D}(i, j)-\mathrm{D}\left(i, j^{\prime}\right)\right) \times\left(p_{i^{\prime}}-p_{i}\right) \\
\geqslant & 0,
\end{aligned}
$$




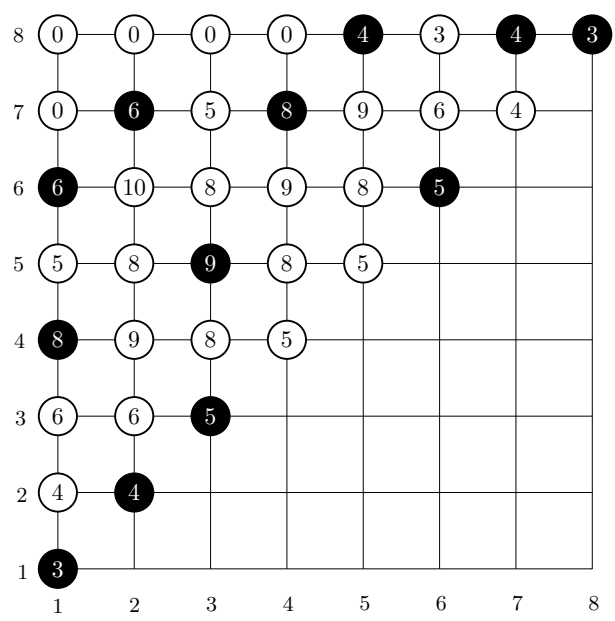

Fig. 4. A refined grid with tokens shown in black. The number on each grid point denotes its magnitude.

where the last inequality holds because $\mathrm{D}\left(i^{\prime}, j^{\prime}\right) \geqslant \mathrm{D}\left(i, j^{\prime}\right), \mathrm{D}(i, j) \geqslant \mathrm{D}\left(i, j^{\prime}\right)$, $p_{i^{\prime}}>p_{i}$, and $q_{j^{\prime}}>q_{j}$.

For a pair of indices $j$ and $j^{\prime}\left(j<j^{\prime}\right)$, we say that a flip occurs between $j$ and $j^{\prime}$ at index $i>1$ if either

$$
\operatorname{GAP}\left(i-1, j, j^{\prime}\right) \geqslant 0 \text { and } \operatorname{GAP}\left(i, j, j^{\prime}\right)<0,
$$

or

$$
\operatorname{GAP}\left(i-1, j, j^{\prime}\right)<0 \text { and } \operatorname{GAP}\left(i, j, j^{\prime}\right) \geqslant 0 .
$$

A flip satisfying the former condition is called a type-1 flip, otherwise it is called a type-2 flip. For example, in the grid shown in Figure 4, there is a type-2 flip between $j=3$ and $j^{\prime}=5$ at $i=2$, and there is a type- 1 flip between $j=5$ and $j^{\prime}=6$ at index $i=3$.

Lemma 3. At any index $i$, a type-1 flip can occur between $j$ and $j^{\prime}\left(j<j^{\prime}\right)$ only if there is a token at $\left(p_{i}, q_{h}\right)$ for some $j \leqslant h<j^{\prime}$.

Proof. This is a direct corollary of Lemma 2.

Our idea for finding a maximum point is to construct a binary tree that maintains the maximum magnitude for the points having the same $x$-coordinate, and update the tree by using flips to find the overall maximum. The algorithm constructs a balanced binary tree $B$ whose leaves are indices $1, \ldots, t$ in increasing order. For an internal node $x$ of $B$, we denote the set of leaves in the subtree rooted at $x$ by $\operatorname{DES}(x)$, the left child of $x$ by $l(x)$ (corresponding to smaller indices), and the right child of $x$ by $r(x)$ (corresponding to larger indices). Note that $\operatorname{DES}(x)$ forms an interval of indices. We denote the largest index among 

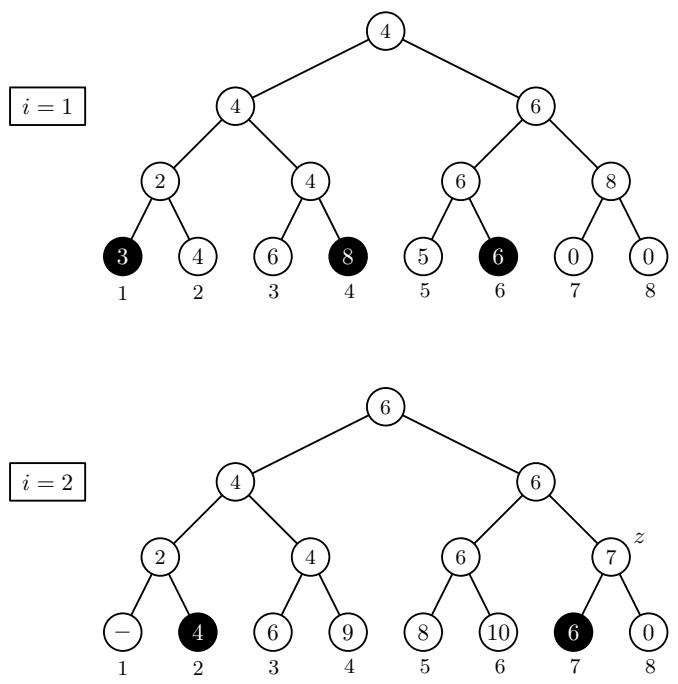

Fig. 5. Binary trees corresponding to the first two columns of the grid depicted in Figure 4 . Numbers in the leaves are magnitudes. For each internal node $x$, the number shown in $x$ is $\mathcal{J}_{i}^{x}$.

$\operatorname{DES}(x)$ by $u(x)$, and the smallest index by $b(x)$. For an index $i$, we define $\mathcal{J}_{i}^{x}$ to be the index $j$ maximizing $\mu(i, j)$ among all indices in $\operatorname{DES}(x)$. Ties are broken by choosing the largest index. See Figure 5 for an illustration. The algorithm iteratively increases $i$ one by one, and at each step $i$, updates $\mathcal{J}_{i}^{x}$ when $\mathcal{J}_{i-1}^{x} \neq \mathcal{J}_{i}^{x}$.

Observation $2 \mathcal{J}_{i-1}^{x} \neq \mathcal{J}_{i}^{x}$ only if either (a) $\mathcal{J}_{i-1}^{l(x)} \neq \mathcal{J}_{i}^{l(x)}$, (b) $\mathcal{J}_{i-1}^{r(x)} \neq \mathcal{J}_{i}^{r(x)}$, or (c) there is a flip between $\mathcal{J}_{i-1}^{l(x)}$ and $\mathcal{J}_{i-1}^{r(x)}$ at index $i$.

By this observation, cases (a) and (b) need a descendant of $x$ which involves case (c). Therefore, to find updates for all nodes in $B$, we only have to find occurrences of case (c). In the example shown in Figure 5 , for the node labeled $z$ we have $\partial_{1}^{z} \neq \mathcal{J}_{2}^{z}$ because there is a type- 1 flip between leaves 7 and 8 at $i=2$. Moreover, for the root of the tree, $r$, we have $J_{1}^{r} \neq J_{2}^{r}$ because a type- 2 flip occurs between leaves 4 and 6 at $i=2$.

In each step $i$, we say that a type- 1 event (resp., a type- 2 event) occurs at a node $x$, if there is type-1 (resp., type-2) flip between $g_{i}^{l(x)}$ and $J_{i}^{r(x)}$ at index $i$. We also say that a type- 0 event occurs at a node $x$ in step $i$ if there is a token at $\left(p_{i}, q_{j}\right)$ for some $\mathfrak{J}_{i}^{l(x)} \leqslant j<\mathcal{J}_{i}^{r(x)}$. By Lemma 3 , any type- 1 event is also a type- 0 event. Therefore, we only need to consider type- 0 and type- 2 events.

To find events in each step efficiently, we keep the earliest coming event for each internal node. More precisely, for each node $x$ and index $i$, we define the next event of $x$ by the smallest index $i^{\prime}$ such that $i^{\prime}>i$ and either a type- 0 or a type-2 event occurs at $x$ in step $i^{\prime}$. If no such index $i^{\prime}$ exists, we say that $x$ has no next flip. In each iteration $i$, we maintain the next event for each internal 
node, and thus, we can find all nodes at which an event occurs in each step by looking only at the next events.

The complete procedure for computing a maximum point is presented in Algorithm 1. In this algorithm, a node $x$ called an update node if an event occurs at some descendant of $x$.

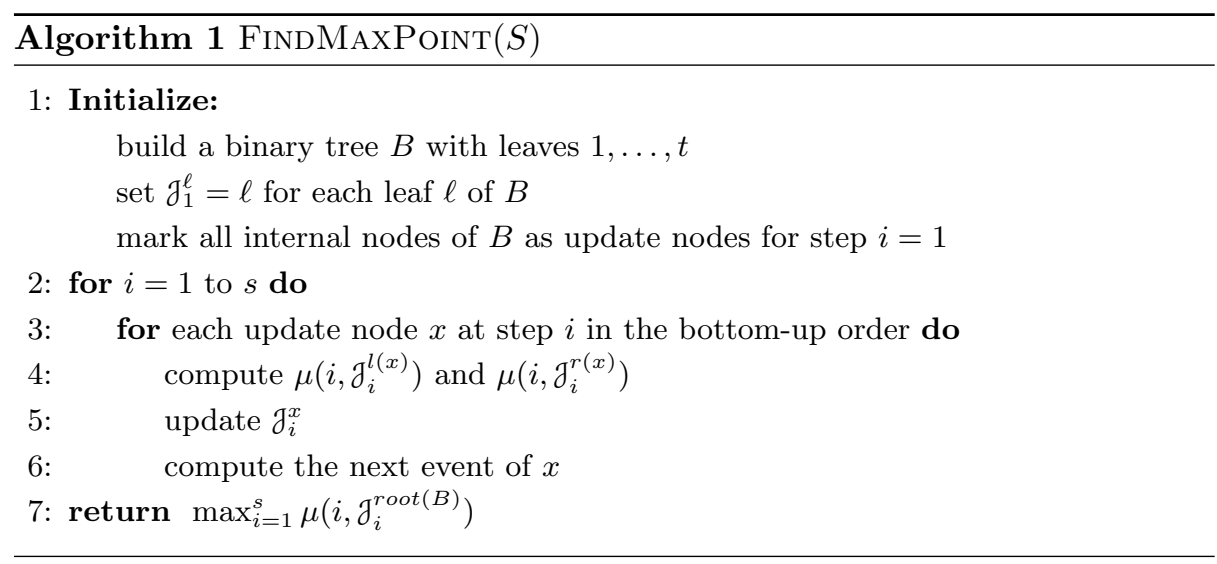

Lemma 4. The total number of update nodes during the execution of Algorithm 1 is $O\left(n \log ^{2} n\right)$.

Proof. The number of ancestors of a leaf is $O(\log n)$, thus the number of type- 0 events is $O(n \log n)$ in total during the execution of the algorithm. We show below that the same bound holds for the number of type- 2 events.

Consider the sequence of events occurring at a node $x$. We show that in this sequence, there is at most one type- 2 event between any two consecutive type- 0 events. Fix a node $x$, and consider two consecutive type- 0 events occurring at $x$, say in steps $a$ and $b$. Since no other type- 0 event occurs in between $a$ and $b$, by Lemma 3 , there is no token at $\left(p_{i}, q_{j}\right)$ for all $a<i<b$ and $b(x) \leqslant j<u(x)$.

Let $c$ be the first step, $a<c<b$, at which a type- 2 event occurs at $x$. We prove that for all subsequent steps $i, c<i<b$, no type-2 event can occur at $x$. The proof is based on the following claim:

Claim. For all $c<i<b, \mu\left(i, g_{i}^{l(x)}\right) \leqslant \mu\left(i, g_{i}^{r(x)}\right)$.

To show this, fix an $i$ such that $c<i<b$. We have

$$
\mu\left(c, \partial_{i}^{l(x)}\right) \leqslant \mu\left(c, \partial_{c}^{l(x)}\right) \leqslant \mu\left(c, \partial_{c}^{r(x)}\right),
$$

where the right-hand inequality holds because a type-2 flip has occurred at $x$ in step $c$, and the left-hand inequality holds because $g_{c}^{l(x)}$ points to a maximum leaf in $\operatorname{DES}(l(x))$ in step $c$. Using (1) and Lemma 2 we get

$$
\mu\left(i, \partial_{i}^{l(x)}\right) \leqslant \mu\left(i, \partial_{c}^{r(x)}\right)
$$


because there is no token at $\left(p_{h}, q_{j}\right)$ for all $c<h \leqslant i$ and $b(x) \leqslant j<u(x)$. Using (2) and the fact that $\mu\left(i, \partial_{c}^{r(x)}\right) \leqslant \mu\left(i, \partial_{i}^{r(x)}\right)$, we obtain the claim's statement.

It thus follows that the number of type- 2 events does not exceed the number of type- 0 events, and therefore, we have $O(n \log n)$ events in total. Since each event can be involved in at most $O(\log n)$ update nodes, the total number of update nodes during the execution of the algorithm is $O\left(n \log ^{2} n\right)$.

Theorem 1. Algorithm 1 solves the maximum point problem for a set of $n$ tokens on a grid in $O\left(n \log ^{3} n \log \log n\right)$ time and $O(n)$ space.

Proof. The correctness of the algorithm follows directly from the fact that at each step $i, g_{i}^{\text {root }(B)}$ maintains the location of a maximum point in column $i$, and therefore, line 7 of the algorithm returns a maximum point in the whole grid. The running time of Algorithm 1 is dominated by the time needed by the two for-loops. The computation of the magnitude $\mu(i, j)$ in line 4 involves computing $\mathrm{D}(i, j)$ which can be done in $O(\log \log n)$ time using an orthogonal range search on the refined grid [13]. For any node $x$, the computation of the next type- 0 event involves finding the earliest coming token in the rectangle $[i . . s] \times\left[\mathcal{g}_{i}^{l(x)} . . J_{i}^{r(x)}\right]$, and the computation of the next type- 2 event involves a binary search on index $i$ with the computation of $O(\log n)$ magnitudes, both can be done in $O(\log n \log \log n)$ time. By Lemma 4, the two for-loops together iterate $O\left(n \log ^{2} n\right)$ times. Therefore, the running time of the algorithm is $O\left(n \log ^{3} n \log \log n\right)$ in total. The binary tree has $O(n)$ nodes and each node requires memory of constant size to keep the maximum index and the next event. The space complexity is therefore $O(n)$. Note that the initialization step involves sorting tokens by their integer coordinates, constructing the binary tree $B$, and initializing the data structure for orthogonal range search, all of which can be done in $O(n)$ time and $O(n)$ space. The proof of the theorem is thus complete.

The next theorem follows directly from Lemma 1 and Theorem 1.

Theorem 2. Given a convex bipartite graph $G=(A, B, E)$, a maximum edge biclique of $G$ can be computed in $O\left(n \log ^{3} n \log \log n\right)$ time and $O(n)$ space, where $n=|A|$.

\section{Algorithms for Special Convex Graphs}

For two subclasses of convex bipartite graphs, namely biconvex graphs and bipartite permutation graphs, we show in this section that the maximum edge biclique problem can be solved efficiently using a simple transformation to a geometric problem for which efficient algorithms are already available.

Biconvex Graphs. A polygon $P$ is called orthogonally convex if every horizontal or vertical segment connecting two points of $P$ lies completely inside $P$. Equivalently, a polygon is orthogonally convex, if it intersects every horizontal 


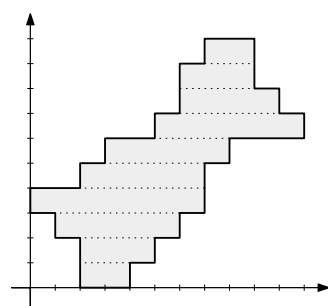

(a)

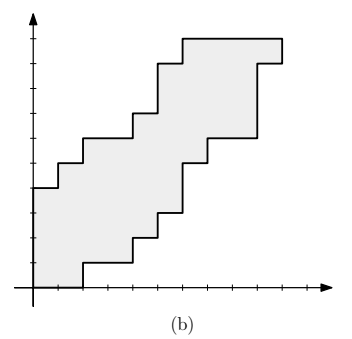

(b)

Fig. 6. (a) An orthogonally convex rectilinear polygon. (b) An $x y$-monotone rectilinear polygon.

or vertical line in at most two points (see Figure 6(a)). As noted in [30], a biconvex graph can be represented as an orthogonally convex rectilinear polygon. The transformation is as follows. Let $G=(A, B, E)$ be a bipartite graph with $A=[1 . . n]$ and $B=[1 . . k]$, where vertices are ordered with respect to the adjacency property. For each edge $(i, j)$, we put a unit square $[j-1, j] \times[i-1, i]$ in the plane. The union of these squares represents a rectilinear polygon that is orthogonally convex. The above transformation takes $O(|E|)$ time. However, since we assume that in the input graph, vertices of $A$ and $B$ are numbered according to the adjacency property, we can perform the transformation faster as follows.

Lemma 5. Given a biconvex graph $G=(A, B, E)$, we can transform $G$ to an orthogonally convex rectilinear polygon of size $O(n)$ in $O(n)$ time and $O(n)$ space, where $n=\min (|A|,|B|)$.

Proof. Suppose w.l.o.g. that $|A| \leqslant|B|$. For $1 \leqslant i \leqslant n$, let vertex $i \in A$ be connected to the vertices $\left[c_{i} . . d_{i}\right]$ in $B$. Starting from an empty polygon $P$, we incrementally add to $P$ a rectangle $R_{i}=\left[c_{i}-1, d_{i}\right] \times[i-1, i]$ for $i$ from 1 to $n$. Updating the polygon at each step involves adding at most 4 vertices to the top of $P$ (see Figure 6(a)), which can be done in constant time. The complete transformation can be therefore carried out in $O(n)$ time.

Next, we show that finding the maximum edge biclique in a biconvex graph is equivalent to the following problem.

Problem 3 (Largest Rectangle). Given an orthogonally convex rectilinear polygon $P$ of size $n$, find the largest area axis-parallel rectangle inside $P$.

Lemma 6. Problem 1 on biconvex graphs is equivalent to Problem 3.

Proof. Let $G=(A, B, E)$ be a biconvex graph, and let $P$ be the polygon obtained from $G$ by the transformation described above. It is easy to observe that any maximal axis-parallel rectangle in $P$ has vertices with integer coordinates. Let $R=\left[i-1, i^{\prime}\right] \times\left[j-1, j^{\prime}\right]$ be an axis-parallel rectangle inside $P$. By our transformation in Lemma 5 , for all $i \leqslant a \leqslant i^{\prime}$ and $j \leqslant b \leqslant j^{\prime}$ we have $(a, b) \in E$, and 
therefore, $R$ corresponds to a biclique $\left[i . . i^{\prime}\right] \times\left[j . . j^{\prime}\right]$ in $G$. On the other hand, if $C=S \times T$ is a biclique in $G$, then by the biconvexity of $G, S$ is an interval $\left[i . . i^{\prime}\right] \subseteq[1 . . n]$ and $T$ is an interval $\left[j . . j^{\prime}\right] \subseteq[1 . . k]$. Therefore, all unit squares $[a-1, a] \times[b-1, b]$ such that $i \leqslant a \leqslant i^{\prime}$ and $j \leqslant b \leqslant j^{\prime}$ are in $P$. The union of these unit squares defines an axis-parallel rectangle $\left[i-1, i^{\prime}\right] \times\left[j-1, j^{\prime}\right]$ inside $P$. Therefore, there is a one-to-one correspondence between bicliques in $G$ and axis-parallel rectangles with integer coordinates in $P$. The theorem statement easily follows by considering the fact that the number of edges in a biclique of $G$ is equal to the area of its corresponding axis-parallel rectangle in $P$.

Daniel et al. [8] showed that the largest area axis-parallel rectangle inside an orthogonally convex rectilinear polygon of size $n$ can be computed in $O(n \alpha(n))$ time and $O(n)$ space, where $\alpha(n)$ is the inverse of the Ackermann function. Combined with Lemmas 5 and 6 , we immediately get the following:

Theorem 3. Given a biconvex graph $G=(A, B, E)$, a maximum edge biclique of $G$ can be computed in $O(n \alpha(n))$ time and $O(n)$ space, where $n=\min (|A|,|B|)$.

Bipartite Permutation Graphs. A rectilinear polygon is called $x y$-monotone if it is monotone with respect to the line $x=y$. Figure 6(b) shows an example of an $x y$-monotone rectilinear polygon. Every $x y$-monotone rectilinear polygon is orthogonally convex, and its boundary consists of only two $x y$-monotone polygonal chains. As noted in [31], a bipartite permutation graph can be transformed to an $x y$-monotone rectilinear polygon, using the same transformation described in the previous section. Using Lemma 5, the transformation takes $O(n)$ time and $O(n)$ space, where $n=\min (|A|,|B|)$. Finding the largest area axis-parallel rectangle inside the resulting $x y$-monotone rectilinear polygon can be done in $O(n)$ time and $(n)$ space using the fast matrix searching algorithm of Aggarwal et al. [1]. The next theorem thus immediately follows.

Theorem 4. Given a bipartite permutation graph $G=(A, B, E)$, a maximum edge biclique of $G$ can be computed in $O(n)$ time and $O(n)$ space, where $n=$ $\min (|A|,|B|)$.

\section{Conclusions}

In this paper, we presented an efficient algorithm for solving the maximum edge biclique problem in convex bipartite graphs in $O\left(n \log ^{3} n \log \log n\right)$ time. The objective function used in our algorithm was $\operatorname{DOM}(i, j) \times(j-i+1)$. The algorithm works as long as the monotonicity of the GAP function is preserved. Therefore, we can generalize the objective function to any arbitrary function of the form $f(\operatorname{DOM}(i, j)) \times g(j-i)$ such that $f$ is monotone increasing in $\operatorname{DOM}(i, j)$, and $g$ is monotone increasing in $j$. For special subclasses of convex bipartite graphs, namely for biconvex graphs and bipartite permutation graphs, faster algorithms are derived as noted in Section 4. 
Some problems remain open. An immediate problem is whether we can improve the running time of the main algorithm presented in Section 3 by removing (some of) the logarithmic factors. Finding a better algorithm for the maximum edge biclique problem in chordal bipartite graphs (which is a direct superclass of convex bipartite graphs) is another interesting open problem.

\section{References}

1. A. Aggarwal, M. Klawe, S. Moran, P. Shor, and R. Wilber. Geometric applications of a matrix-searching algorithm. Algorithmica, 2(1-4):195-208, 1987.

2. G. Alexe, S. Alexe, Y. Crama, S. Foldes, P. L. Hammer, and B. Simeone. Consensus algorithms for the generation of all maximal bicliques. Discrete Appl. Math., 145(1):11-21, 2004.

3. A. Ben-Dor, B. Chor, R. Karp, and Z. Yakhini. Discovering local structure in gene expression data: the order-preserving submatrix problem. J. Comput. Biol., 10(3-4):373-384, 2003.

4. K. S. Booth and G. S. Lueker. Testing for the consecutive ones property, interval graphs, and graph planarity using PQ-tree algorithms. J. Comput. Systems Sci., 13(3):335-379, 1976.

5. A. Brandstädt, V. B. Le, and J. P. Spinrad. Graph classes: a survey. SIAM, Philadelphia, 1999.

6. G. Brodal, L. Georgiadis, K. Hansen, and I. Katriel. Dynamic matchings in convex bipartite graphs. In Proc. 32nd Internat. Sympos. Math. Found. Comput. Sci., pages 406-417, 2007.

7. Y. Chen and G. Church. Biclustering of expression data. In Proc. 8th Internat. Conf. Intelligent Systems for Molecular Biology, pages 93-103, 2000.

8. K. Daniels, V. Milenkovic, and D. Roth. Finding the largest area axis-parallel rectangle in a polygon. Comput. Geom. Theory Appl., 7(1-2):125-148, 1997.

9. M. Dawande, P. Keskinocak, J. M. Swaminathan, and S. Tayur. On bipartite and multipartite clique problems. J. Algorithms, 41(2):388-403, 2001.

10. V. M. Dias, C. M. de Figueiredo, and J. L. Szwarcfiter. Generating bicliques of a graph in lexicographic order. Theoret. Comput. Sci., 337(1-3):240-248, 2005.

11. V. M. Dias, C. M. de Figueiredo, and J. L. Szwarcfiter. On the generation of bicliques of a graph. Discrete Appl. Math., 155(14):1826-1832, 2007.

12. U. Feige. Relations between average case complexity and approximation complexity. In Proc. 34th Annu. ACM Sympos. Theory Comput., pages 534-543, 2002.

13. O. Fries, K. Mehlhorn, S. Näher, and A. Tsakalidis. A $\log \log n$ data structure for three-sided range queries. Inform. Process. Lett., 25(4):269-273, 1987.

14. B. Ganter and R. Wille. Formal Concept Analysis, Mathematical Foundations. Springer-Verlag, Berlin, 1996.

15. A. Gély, L. Nourine, and B. Sadi. Enumeration aspects of maximal cliques and bicliques. Discrete Appl. Math., 157(7):1447-1459, 2009.

16. F. Glover. Maximum matching in a convex bipartite graph. Naval Res. Logist., 14:313-316, 1967.

17. A. Goerdt and A. Lanka. An approximation hardness result for bipartite clique. In Technical Report 48, Electronic Colloquium on Computation Complexity. 2004.

18. M. Habib, R. McConnell, C. Paul, and L. Viennot. Lex-BFS and partition refinement, with applications to transitive orientation, interval graph recognition and consecutive ones testing. Theoret. Comput. Sci., 234(1-2):59-84, 2000. 
19. T. Kloks and D. Kratsch. Computing a perfect edge without vertex elimination ordering of a chordal bipartite graph. Inform. Process. Lett., 55(1):11-16, 1995.

20. Y. D. Liang and M. Chang. Minimum feedback vertex sets in cocomparability graphs and convex bipartite graphs. Acta Inform., 34(5):337-346, 1997.

21. W. Lipski and F. P. Preparata. Efficient algorithms for finding maximum matchings in convex bipartite graphs and related problems. Acta Inform., 15(4):329-346, 1981.

22. S. C. Madeira and A. L. Oliveira. Biclustering algorithms for biological data analysis: A survey. IEEE ACM Trans. Comput. Biol. Bioinform., 1(1):24-45, 2004.

23. J. Meidanis, O. Porto, and G. P. Telles. On the consecutive ones property. Discrete Appl. Math., 88(1-3):325-354, 1998.

24. N. Mishra, D. Ron, and R. Swaminathan. On finding large conjunctive clusters. In Proc. 16th Annu. Conf. Computational Learning Theory, pages 448-462, 2003.

25. R. Peeters. The maximum edge biclique problem is NP-complete. Discrete Appl. Math., 131(3):651-654, 2003.

26. J. Soares and M. Stefanes. Algorithms for maximum independent set in convex bipartite graphs. Algorithmica, 53(1):35-49, 2009.

27. G. Steiner and J. S. Yeomans. A linear time algorithm for maximum matchings in convex, bipartite graphs. Comput. Math. Appl., 31(12):91-96, 1996.

28. J. Tan. Inapproximability of maximum weighted edge biclique and its applications. In Theory and Applications of Models of Computation, pages 282-293. 2008.

29. A. Tanay, R. Sharan, and R. Shamir. Discovering statistically significant biclusters in gene expression data. Bioinformatics, 18(Supplement 1):S136-S144, 2002.

30. C. Yu and G. Chen. Efficient parallel algorithms for doubly convex-bipartite graphs. Theoret. Comput. Sci., 147(1-2):249-265, 1995.

31. C. Yu and G. Chen. An efficient parallel recognition algorithm for bipartitepermutation graphs. IEEE Trans. Parallel Dist. Sys., 7(1):3-10, 1996. 